\title{
FUNDAMENTAL PARAMETERS AND THE HELIUM CONTENT OF ECLIPSING SPECTROSCOPIC BINARIES
}

\author{
H. E. Jørgensen \\ Copenhagen Observatory
}

Today we know accurate masses, radii, surface gravities and luminosities or effective temperatures of a very small number of stars. There are several reasons for deriving accurate parameters of stars. I shall mention only four problems:

(i) checking stellar evolution calculations, particularly calculations of isochrones, using binaries,

(ii) derivation of helium content in stars of different age,

(iii) checking log g derived from photometry and stellar atmosphere calculations,

(iv) determination of accurate positions in the HR diagram to get an independent check on calibrations of photometric systems.

At the Copenhagen University Observatory we have carried out a program deriving fundamental parameters of eclipsing spectroscopic binaries. The spectroscopy is done by $\mathrm{J}$. Andersen with the ESO $1.5 \mathrm{~m}$ spectrographic telescope at La Silla (in some cases in collaboration with D.M. Popper). The photometry is done by a number of people in Copenhagen using our $50 \mathrm{~cm}$ reflector at La Silla. Our telescope at La Silla is equipped with a simultaneous four channel spectrographic photometer.

The four channels correspond to the Strömgren uvby photometric system. At present we have obtained approximately 40,000 accurate photometric observations of selected eclipsing binaries.

Our philosophy in this program is to study binaries with well separated components to avoid complications such as gas in the 
system, strong deformation of the components, strong reflection effects, etc. Models of binaries with such complications are not very well developed and derived parameters of stars in such systems are therefore not very accurate. A binary with well separated components can be described using a relatively simple model. We use the model by Wood (1971).

Let us now ask the questions: What accuracy must we have to solve the problems (i) - (iv) already mentioned? We shall particularly consider the helium content problem. From measurements of emission lines from $H$ II regions in the optical and radio region a present helium content in the interstellar medium is derived. From accurate photoelectric measurements of the helium line at $4026 \AA$ Nissen (1976) has derived helium abundances in atmospheres of $B$ stars. Both the emission line measurements and the $4026 \AA$ measurements in the young stars seem to indicate a variation in the helium abundance from place to place. The abundance is normally found to be in the range $Y=0.20-0.30$ and the variation from place to place amounts approximately to $\Delta \mathrm{Y}=0.10$. Also for very old objects such as globular clusters and halo planetary nebulae we may give an estimate of helium abundance. However the abundance of helium is, in this case, derived from stars in late evolutionary stages and we are ususally left with both the uncertainty of enrichment in the envelopes and the uncertainty in internal structure of horizontal-branch stars. Values in the range $Y=0.2-0.4$ are often obtained.

At present we have little evidence for any general increase in helium abundance with time, although it has been proposed that enrichment of helium in the interstellar medium is correlated with the enrichment by heavier elements.

From this discussion it is obvious that the expected range in the abundance of helium from star to star is no more than $\triangle Y \sim 0.10$. To obtain further knowledge of an eventual enrichment of the interstellar medium we must therefore determine the helium abundance with an accuracy of approximately $\Sigma(Y)=0.03$. From stellar evolution calculations we can easily estimate that this corresponds to a typical set of uncertainties in the fundamental parameters: 2 per cent in mass, 1 per cent in radius, 2 per cent in effective temperature and 25 per cent in $\mathrm{Z}$, the content of heavier elements.

Our results are given in Table $I$. The masses are in most cases determined to an accuracy of 2 per cent and the radii are known with sufficient precision. To derive effective temperatures we use $[u-b]$ for $B$ stars and the $\beta$ index for the late $A$ and $F$ stars. In a few cases we still have no $\beta$ index available and use (b-y), since the star is nearby. The $\mathrm{m}_{1}$ index is used to determine $[\mathrm{Fe} / \mathrm{H}]$ and from that the value of $Z$. An accuracy of 25 per cent in $Z$ corresponds to an accuracy of 0.007 in $\mathrm{m}_{l}$, which normally is no problem. 


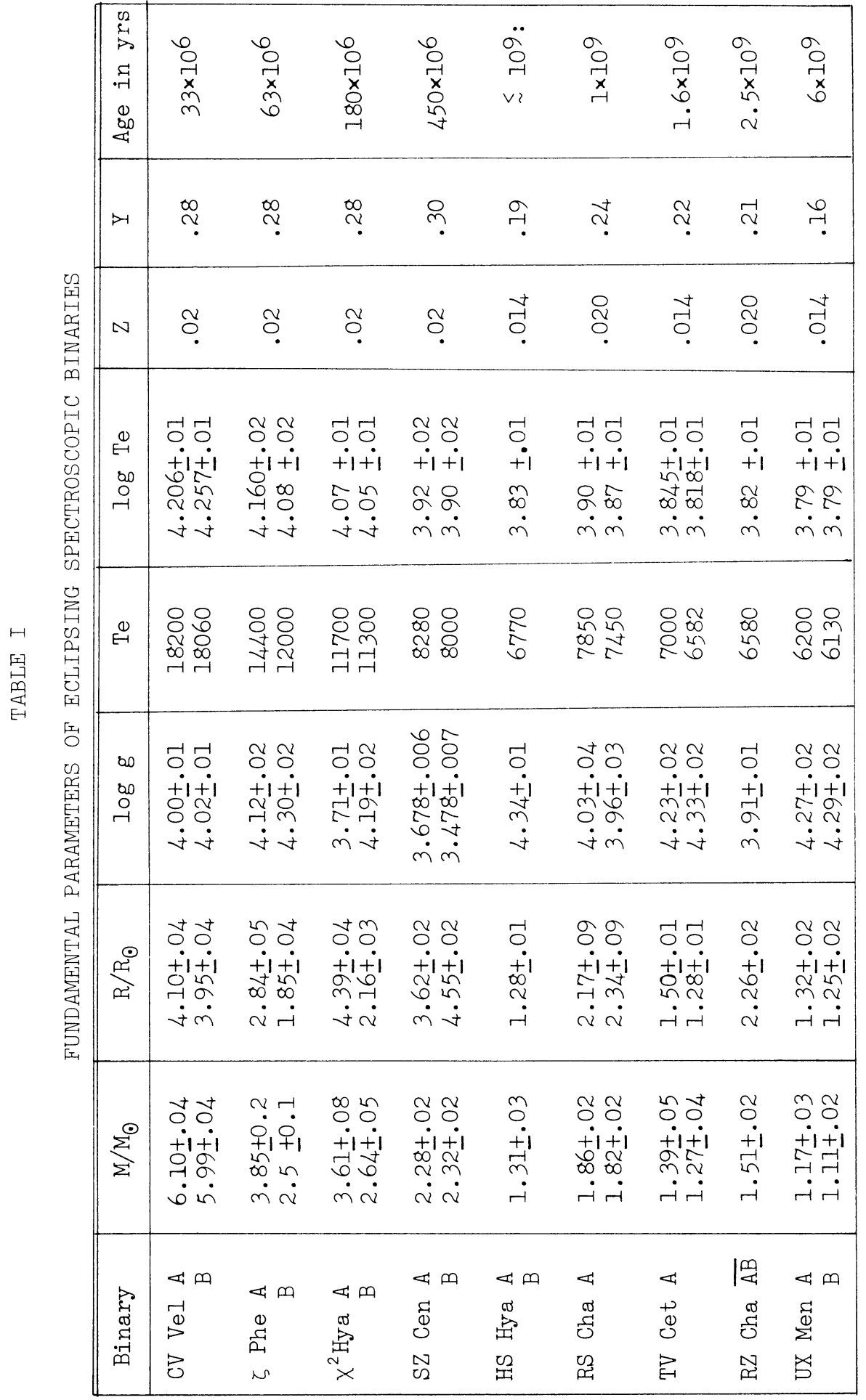


The derived helium abundances in Table I are very close to 0.28 for the B stars and close to 0.22 for the F stars. Half of this difference (0.03) could be explained by the fact that $\bar{Z}$ for the F stars equals 0.016 and not 0.020 as adopted for the B stars. The other half $(0.03)$ is most easily understood as a small error in the temperature scale or by errors in the calculated opacities. We notice, in particularly, the small variation in the helium content of $F$ stars, where the spread is not more than $\Delta \mathrm{Y}=0.03$ around the mean value of $\mathrm{Y}=0.22$.

\section{REFERENCES}

Nissen, P.E. (1976). Astron. Astrophys. 50, 343. Wood, D.B. (1971). Astron. J. 76, 701. 